\title{
THE RELATIONSHIP BETWEEN OBESITY AND OVERWEIGHT WITH THE INTAKE OF VITAMIN D AND CALCIUM RICH FOODS AMONG PRIVATE UNIVERSITY STUDENTS IN SHAH ALAM, MALAYSIA
}

\author{
Tiba Nezar Hasan*, Puteri Farrah Nadhira Radhi \\ Corresponding author:dr.tibahasan@gmail.com
}

International Medical School, Management and Science University, Selangor, Malaysia

\begin{abstract}
Introduction: Obesity and overweight are one of the health problems that critically increasing nowadays in worldwide including Malaysia due to lifestyle changes such as inactive lifestyle as well as unhealthy eating habit. This study, therefore is to find if there is a relationship between obesity and overweight with the intake of vitamin $D$ and calcium rich foods to see if there is any difference intake between normal, obesity and overweight group. Methods: A cross-sectional study was conducted among 255 Management and Science University students (MSU) who having a normal, overweight and obesity of body mass index. Data was collected using self-administered questionnaire consisting of two part which is sociodemographic questions and Food Frequency Questionnaire (FFQ).

Result: For calcium intake, there is a significant association between body mass index (BMI) and calcium which is $(p=0.008)$ respectively and there is no significant association between BMI and Vitamin D intake $(p=0.594)$ Conclusion: The study found that there is a relationships between obesity and overweight with intake of calcium rich food and no relationship between obesity and overweight with the intake of Vitamin D. Further study are needed to build causal relationships and develop preventative measures.
\end{abstract}

Keywords: Obesity, Overweight, Vitamin D, Calcium, Students, Malaysia 


\section{Introduction}

Obesity results from consuming food that give high energy and calories that exceed the body needs in addition to low physical activity over long time (Hill et al. 2010; Daniels et al. 2005). During the growing years, the main causes of obesity are different with many factors, some of these factors are nonmodifiable and some are modifiable factors from the environment (Lobstein et al. 2004; Hill et al. 2003) and can sometimes worsen by biological growth factors during the rapid growth spurt at the time of puberty(Siervogel et al. 2003). The most effective way of reducing weight or prevent weight gain come through healthy life style(Daniels et al. 2005; Hills et al. 2010), the practices of sedentary lifestyle and less physical activity such as watching television, computer and media used have high effect on obesity risk among populations in Malaysia (Epstein et al. 2008; Sugiyama et al. 2008).

BMI can be calculated by dividing a person's weight in kilograms by the square of a person's height in meters. The category of overweight and obesity is derive from data gathered from population-based epidemiology studies that examined the relationship between obesity and rates of mortality and morbidity that are adiposity related. It can be considered overweight if the BMI $(\mathrm{kg} / \mathrm{m} 2)$ is between 25 and 29.9. Obesity is defined when the BMI is $\geq 30$ and can be further classified into three classes beginning from class I till class III. There is evidence to shows the risks of adiposity-related complications occur at lower BMIs in Asians. Therefore, for obesity, China used a BMI of 28 and Japan used a BMI cut-off of $25 \mathrm{~kg} / \mathrm{m} 2$ for cut-off. The World Health Organization (WHO) has recommended that $\mathrm{BMI}>27.5$ $\mathrm{kg} / \mathrm{m} 2$ be used as a cut off for Asians, considering the increased risk of cardiovascular system problem at the BMI (Fock et al. 2013).

Obesity is not considered as one of the psychiatric disorders. However, people who have a psychiatric disorder such as depression have a high risk of being obese. This increase in weight can be due to some medications such as antidepressants, antipsychotic medications antidiabetic drugs, anticonvulsants, beta blockers in addition to steroid hormones. Weight gain also can be seen in people who quit smoking. It is also essential to note comorbidities associated with obesity including diabetes mellitus, high lipids profile, high blood pressure, and cardiovascular disease (Fock et al. 2013).

The basis of therapy for losing weight is dietary intervention and planning. It is suggested from most of the dietary regimens to have more concern on the content of energy and compositions of macronutrients in order to reduce weight. This energy content mainly affects the result and effectiveness of the dietary plans. The guidelines of obesity treatment that issued by the National Institute Health recommended that those who are overweight or with class I obesity with two or more risk factors should reduce their daily energy intake by $500 \mathrm{kcal}$ while those with class II and class III obesity must reduce daily between 500-1000 kcal. Weight loss of about $0.5 \mathrm{~kg} / \mathrm{week}$ will result from reducing this 500 $\mathrm{kcal} /$ day of energy intake. It is crucial to calculate the daily requirement of energy for patient to give them diet that have lower energy and lead to loss of weight, this can be calculated using the Harris Benedict equation or the World Health Organ equation or American Gastroenterological Association dietary guidelines (Klein et al. 2002).

Therefore, the aim of this study is to find the relationship between obesity and overweight with the intake of vitamin $D$ and foods rich in calcium to see if there is any difference intake between normal, obesity and overweight group.

\section{Methods}

The study design that have been used is cross sectional studies that was carry out to find the relationship between overweight and obesity with calcium and vitamin D intake among Management and Science University students in Shah Alam, Selangor. The study was conducted in Management and Science University, Shah Alam, Selangor. The study population was the students of Management and Science University in Shah Alam, Selangor.

Sample was collected through convenience method. The respondents consist of male and female students aged 18 years and above. There are 255 respondents selected which is 85 have a normal body mass index (18.524.9), 85 have overweight body mass index (25-29.9) and 85 have Obesity body mass index $(\geq 30)$. Those who refuse to participate in the research was exclude from the study. 
Data collection was done by using selfadministered questionnaires. It consists of two sections including part $A$ sociodemographic details and part B Food Frequency Questionnaire (FFQ). All respondent need to recall back their dietary meal in one week. Weight scale and stadiometer is used to measure the weight and height to get the BMI.

The nutrition data was calculated by using a software called 'Nutritionist Pro' to get the total intake of calcium and vitamin D in one week. The collected data is entered and have been analyses by using SPSS version 25 . One-way Anova and T- test is used to determine the association between obesity and overweight with the intake of vitamin $D$ and calcium rich foods.

All participants need signed the consent for before answering the questionnaires and full explanation of the study was given to them prior to enrolment.

\section{Results}

A total of 255 students participate in this study or research which is 85 of students have a normal body mass index (BMI), 85 of students have an overweight BMI and the other 85 students have an obesity BMI. For the normal BMI, the mean intake of vitamin $D$ is 18.83 , overweight 23.68 and obesity is 25.68. The results shows that the highest average for the total intake of vitamin $D$ is in obesity group which is 25.68 .

The minimum level of vitamin $D$ intake for all group is .00. However, for the maximum level there is different between normal, overweight and obesity. The maximum level for vitamin D intake in a normal BMI is 97.41 , overweight is 88.14 and obesity is 614.27 .

For the calcium intake, the mean intake for a normal BMI is 6192.25 , overweight is 5928.64 and obesity is 4791.34 . The highest average for the total intake of calcium is in a normal group which is 6192.25 .

The minimum level of calcium intake for a normal BMI is 1074.55, overweight is 1005.14 and for obesity is 736.04 . For the maximum level in normal, the range is 22180.49, overweight is 23632.72 and obesity is 15094.38 .
Results shows the association between BMI with vitamin $D$ and calcium intake. The $F$ score for the vitamin $D$ is .522 and the significant level is .594. It shows that there is no significant between the BMI groups (normal, overweight and obesity) with the intake of vitamin $\mathrm{D}$.

The F score for calcium intake is 4.986 and the significant level is .008 . It indicates that there is a significant level between the BMI groups (normal, overweight and obesity) with the intake of calcium.

To be concluded, there is an association between obesity and overweight with the intake of calcium but there is no association between obesity and overweight with the intake of vitamin $D$ in foods. This can be proved based on the $p$ value which is $<0.05$ $(p=0.008)$ that is means there is a significant in the calcium intake with obesity and overweight. However, for vitamin $D$, the $p$ value is $>0.05$ which is $p=$ .594. It indicates that there is no significant for the vitamin $d$ intake with obesity and overweight.

Table1 Vitamin D and calcium intake among male and female students

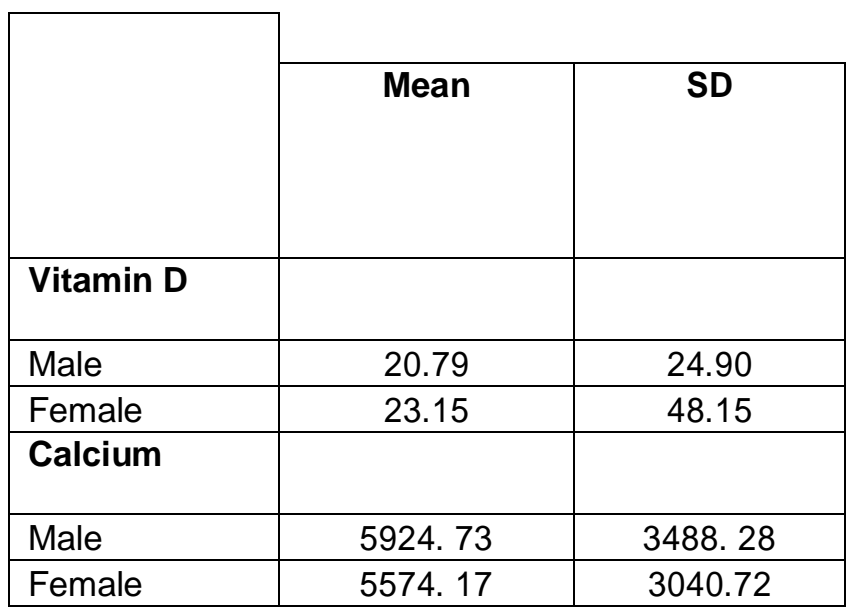

\section{Discussion}

The main finding of our current study is that high vitamin $\mathrm{D}$ intake among the $\mathrm{BMI}$ is 25.68 which in obesity group. The maximum intake of vitamin D in obesity group is 614.27 . This results indicates that people with obesity BMI consumed more vitamin $D$ in their meals compared with people who having normal and overweight BMI. 
For the calcium intake, the high average is under a normal group of BMI which is 6192.26. However, the maximum calcium intake is 23632.72 which is under overweight group.

The highest intake of calcium in the normal group is slightly less than those overweight group which is only 22180.49. Both results for vitamin $\mathrm{D}$ and calcium have support from the previous research. In the population of northern Norway, significant and positive association was founded between calcium intake and body mass index (BMI) in men, and negative significant associations were found between the intake of vitamin $\mathrm{D}$ and $\mathrm{BMI}$ in both sexes (Kamycheva et al. 2004).

Results shows that the group statistics of male and female along with the BMI and the intake of vitamin $D$ and calcium. The total of female respondents is high compared with male which is female 209 and male is 46. The total average for vitamin $D$ intake in female is higher than male where is the total average for calcium intake in female is lower than male.

There is no association between gender male and female with vitamin $D$ and calcium intake. There have been previous human studies (Zemel et al. 2000; Carruth at al. 2001; Heaney et al. 2003) that studied the association between dietary calcium and body weight and fat. In previous research by (Zemel et al. 2003) examined epidemiological National Health and Nutrition Examination Survey III data and revealed that after controlling for energy intake, the relative risk of being in the highest quartile of body fat was the highest for those consuming diets lowest in calcium. This reverse relationship between body fat and calcium intake was found for both men and women.

\section{Conclusion}

This study was conducted to find out if there is a relationship between obesity and overweight with the intake of vitamin D and foods rich in calcium among MSU students. A group of normal, overweight and obesity body mass index consists of male and female is randomly selected to answer the Food Frequency Questionnaire. The findings of the study showed that there is relationship or association between the BMI with the calcium intake. However, there is no association between the BMI with the vitamin D intake. is rejected for the calcium intake but the null hypothesis is accepted for the vitamin $\mathrm{D}$ intake. To be concludes, people is recommended to consume more calcium in their dietary meals as one of the prevention of obesity and overweight.

\section{References}

Daniels SR, Arnett DK, Eckel RH, Gidding SS, Hayman LL, Kumanyika S, et al. (2005) Overweight in children and adolescents: pathophysiology, consequences, prevention, and treatment. Circulation;111:1999-2012.

Carruth BR, S. J. (2001). The role of dietary calcium and other nutrients in moderating body fat in preschool children. Int $J$ Obes Relat Metab Disord,25(4), 559-566.

Epstein LH1, R. J., Robinson JL, Paluch RA, Winiewicz DD, Fuerch $\mathrm{JH}$, \& Robinson TN. (2008). A randomized trial of the effects of reducing television viewing and computer use on body mass index in young children. Arch Pediatr Adolesc Med, 162(3), 239-245. doi:10.1001/archpediatrics.2007.45

Fock KM, K. J. (2013). Diet and exercise in management of obesity and overweight. Journal of Gastroenterol and Hepatology, 4, 59-63. doi:10.1111/jgh.12407

Heaney RP, Dowell MS, Hale CA ,Bendich A (2003)Calcium absorption varies within the reference range for serum 25 hydroxyvitamin D.J Am Coll Nutr22: 142146

Hill JO, W. H., Reed GW, \& Peters JC. (2003). Obesity and the environment: Where do we go from here? Science, 299(5608),853-855. doi:10.1126/science. 1079857

Hills AP, O. A., \& Baur LA. (2010). Addressing childhood obesity through increased physical activity. Nat Rev Endocrinol, 6(10), 543-549. doi:10.1038/nrendo.2010.133

Kamycheva E, S. J., \& Jorde R. (2004). Serum parathyroid hormone level is associated with body mass index. Eur J Endocrino, 151(2), 167172.
There is also no association between gender for each of BMI with the vitamin $D$ and calcium intake. The null hypothesis 
Klein S, Wadden T, Sugerman HJ. (2002) AGA technical review on obesity. Gastroenterology. ; 123: 882 - 932.

Lobstein T1, B. L., \& Uauy R; IASO International Obesity TaskForce. (2004). Obesity in children and young people: A crisis in public health. 1, 4104. doi:10.1111/j.1467-789X.2004.00133.x

Siervogel, RM, Demerath, EW, Schubert, C, et al. (2003) Puberty and body composition. Horm Res. 2003; 60 (suppl 1): $36-45$.

Sugiyama T, H. G., Dunstan DW, Salmon J, \& Owen N. (2008). Is television viewing time a marker of a broader pattern of sedentary behavior? Ann Behav Med, 35(2), 245-250. doi:10.1007/s12160-008-9017-z

Zemel MB, S. H., Greer B, Dirienzo D, \& Zemel PC. (2000). Regulation of adiposity by dietary calcium. FASEB J, 14(9), 1132-1138. 